\title{
La formación del ciudadano como producto de la universidad pública en tiempo internacional neoliberal, en la ciudad de Monterrey Nuevo León, Méxicor
}

\section{The formation of the citizen as a product of the public university on neoliberal international time, in the city of Monterrey Nuevo Leon, Mexico}

\author{
Jorge Leal Iga ${ }^{2}$ \\ Universidad Autónoma de Nuevo León, México \\ jorge.lealig@uanl.edu.mx \\ https://orcid.org/0000-0002-5548-1943
}

Recibido: 08/06/2020 - Aceptado: 29/12/2020 - Publicado: 17/02/2021

\begin{abstract}
RESUMEN
Debido a la tendencia internacional neoliberal, las instituciones educativas de nivel público superior en el mundo han modificado su accionar con el fin de adaptarse a las nuevas formas estipuladas por los organismos de financiamiento económico internacional, transformando la formación académica de lo que solía representar la educación pública en el mundo. La presente investigación intentó encontrar si la formación de los estudiantes de universidad pública en la actualidad muestra una tendencia al perfil individualista que privilegia la satisfacción de su interés personal antes que los intereses de la comunidad, lo cual es característico de la ciudadanía neoliberal. Se contó con la participación total de 379 sujetos en dos muestras para
\end{abstract}

(C) Los autores. Este artículo es publicado por Pensamiento Crítico de la Facultad de Ciencias Económicas, Universidad Nacional Mayor de San Marcos. Este es un artículo de acceso abierto, distribuido bajo los términos de la licencia Creative Commons Atribucion - No Comercia_Compartir Igual 4.0 Internacional. (http://creativecommons.org/licenses/by-nc-sa/4.0/) que permite el uso no comercial, distribución y reproducción en cualquier medio, siempre que la obra original sea debidamente citada. 
lograr tal objetivo, con los que se determinó que, aunque no existe esta formación neoliberal de manera generalizada en los grupos analizados, si se detectó una tendencia particularizada en perfiles determinados e incongruencias en el comportamiento de dichos perfiles.

Palabras clave: Ciudadanía; neoliberalismo; humanitarismo; universidad pública; estudiante universitario; formación académica.

JEL: I23

\section{ABSTRACT}

Due to the neoliberal international trend, the public higher educational institutions in the world have modified their actions in order to adapt themselves to the new ways stipulated by the international economic financing organizations, transforming the academic forming of what the public education in the world used to represent. This investigation tried to find out if the formation of the current public university students shows a tendency towards the individualistic profile that privileges the satisfaction of their personal interest before the interests of the community, which is characteristic of the neoliberal citizenship. There was a total participation of 379 people in two samples to achieve this objective, with which it was determined that although this neoliberal formation does not exist in a generalized way in the groups analyzed, there is indeed a particular tendency that was detected in certain profiles, as well as inconsistencies in the behavior of the profiles described.

Keywords: Citizenship; neoliberalism; humanitarianism; public university; college student; academic training.

JEL: I23 


\section{Introducción}

La universidad representa la memoria colectiva de las ideas de quienes la integran en conjunto con la sociedad misma, producto del debate libre y racional que lamentablemente a causa del enfoque institucional global, se encuentra actualmente en decaimiento (Brunner, 2015, pp. 98, $100 \mathrm{y}$ 108). Las tecnologías de información actuales en el sector productivo y el surgimiento de necesidades propias del trabajo, han promovido nuevos esquemas de competencia (Salazar, 2018, p. 55), lo cual impulsó a las universidades a la adaptación de sus programas académicos acorde a la situación que exige el mercado, sin advertir que aunque las tecnologías de información son un camino para la aplicación en el ámbito laboral, no significan en sí un formato pedagógico de aprendizaje (Massé, 2008, pp. 390-301).

La rígida vinculación que exigen las empresas a las universidades públicas las ha llevado a la necesidad institucional de cancelar programas educativos, tales como la carrera de Filosofía por considerarla sin demanda en el mercado, incluso en la acreditación de becas para jóvenes que deberán seleccionar ahora su vocación académica de acuerdo con las actividades funcionales de las empresas donde pretenden ser contratados (Ornelas, 2009, pp. 90-91 y 105).

La educación universitaria desde el enfoque neoliberal ha tendido a transformar los títulos académicos en un producto mercantil (Fernández, 2017, p. 5) convirtiendo a los ciudadanos con derechos educativos legítimos, a nivel de consumidores (Miñana y Rodríguez, 2002, pp.10 y 15) que ahora intentan conseguir un documento válido únicamente para facilitar su incursión laboral; como una forma de mercantilizar el conocimiento (Burke, 2002; Massé, 2008, p. 389).

Desde el enfoque neoliberal, la educación académica depende del ámbito privado por lo que no tiene que ver con la educación sino con la instrucción ejecutiva, denominada competencia (Vázquez, 2015, pp. 97-98 y 101), esta determinación aspira a lograr un Estado mínimo, en donde se espera que la privatización de la educación promueva su supuesta eficiencia en el esquema de la Nueva Gestión Pública [NGP] (Fernández, 2017, p. 12), enfoque que rechaza a la educación pública porque según su principio, significa un monopolio que depende del recurso público e inhibe la 
participación eficaz de los competidores en el mercado de la educación, en una visión que no considera el efecto social que representa la educación pública superior (Ornelas, 2009, pp. 89-90 y 94).

Dados estos motivos, la universidad pública requiere replantearse desde la perspectiva neoliberal como una empresa de servicios educativos (Miñana y Rodríguez, 2002, p. 7), que se encuentra en competencia con otras del sector e incluso requiere conseguir clientes vinculados a las empresas para promover ingresos externos como recurso para su subsistencia autofinanciable, orientándose a la investigación aplicada en lugar de la ciencia básica, dejando sin atención al pensamiento crítico en la academia (Ornelas, 2009, pp. 86, 98, 100-101). Instituciones como el Banco Mundial, han declarado que la universidad pública especialmente en Latinoamérica es dependiente en exceso del subsidio del Estado, por lo que advierten de la necesidad del control salarial de los docentes entre otras acciones para su justificación económica (De la Cruz, 2012, pp. 50-51).

En la década de los años ochenta, mediante el concepto de la Nueva Administración Pública, se promovieron reformas en la función del Estado en materia de educación superior, que re-direccionaron los alcances académicos establecidos (Bernasconi, 2014, p. 14) para transformarse más en un asunto mercadológico que pedagógico (Miñana y Rodríguez, 2002, p. 15) y para el año 1990 las universidades fueron instruidas con motivo de adaptar a sus estudiantes a la demanda laboral, provocando la formación de individuos egoístas y utilitaristas, en seguimiento a las políticas neoliberales y en detrimento de la institucionalización académica de la universidad como tal (De la Cruz, 2012, pp. 49 y 52), medidas sugeridas incluso por instituciones como la Organización para la Cooperación y el Desarrollo Económico [OCDE] (Ornelas, 2009, p. 97).

En México, esta afectación promovió la pérdida de autonomía en materia de educación pública, dados los dictados para la nueva política educativa proveniente de instituciones tales como el Fondo Monetario Internacional [FMI] y El Banco Mundial [BM] (Salazar, 2018, pp. 57-58). Los organismos internacionales mencionados, consideran de mayor importancia a la eficiencia laboral que a la formación académica de ciudadanos producto de la universidad pública y su compromiso con la sociedad, por lo que esta investigación abordará su precisión. 
Horrach (2009) advierte que el concepto de ciudadanía se construye desde la interrelación del individuo y su medio social, que, debido a su necesidad de expresión en libertad, también se encuentra relacionado directamente con el concepto de democracia (Horrach, 2009, p. 2), pero la dirección de las instituciones y políticas neoliberales le infieren a la universidad pública, el objetivo opuesto a sus originales concepciones de ciudadanía con enfoque en la colectividad.

Derivado del concepto genérico de modelos de ciudadanía según Horrach (2009, pp. 14-16), los alcances institucionales producto de la política económica, dirigen hacia la construcción de un modelo de ciudadanía liberal, donde el bien individual se persigue por encima del bien común y se espera que no intervenga el Estado como agente activo, mientras que el objetivo institucional de la universidad pública ha sido el de seguir un modelo de ciudadanía comunitarista, donde se privilegia el interés común antes del individual, con la firme intención de preservar los valores y la participación en asuntos políticos, en el que el Estado funciona como un agente de conservación comunitaria.

Ortiz (2014), destaca un modelo presente en la actualidad que denomina como ciudadanía neoliberal, el cual debe su formación al nuevo paradigma global que concibe: al Estado, al mercado y a la sociedad, como un proyecto civilizatorio que requiere para su cumplimiento de un cierto tipo de perfil del ciudadano pues se modifica su condición legal, así como su participación, ya que en este modelo, el Estado no se obliga a garantizar los derechos ciudadanos incluidos dentro de la noción de justicia social, en el entendido de que es el propio ciudadano el encargado de sí mismo; desde esta apreciación del mercado sin regulación del Estado, se modifican las formas de inserción en lo laboral y las relaciones sociales para el ciudadano.

Por todo lo aquí mencionado, se aprecia que la línea de influencia neoliberal marcada por las instituciones internacionales incita a la universidad pública en la formación de un modelo de ciudadanía liberal o neoliberal para sus estudiantes, en el que se destaca que en la instrucción del alumno se interponga el individualismo preponderantemente sobre el interés comunitario. Pero el paso cronológico en la vida de un estudiante universitario se presenta precisamente en el proceso de su etapa de transición a la edad adulta, y según los artículos 34 y 45 de la Constitución 
Política de los Estados Unidos Mexicanos, se considera ciudadano de la nación, a todo hombre o mujer mexicano(a) mayor de 18 años de edad cumplidos, con derecho a formar parte libremente de los asuntos políticos de su país en forma pacífica (Orden Jurídico Nacional, 2019), por lo que el estudiante de nivel superior como ciudadano, tiene derecho a participar y opinar sobre la vida política de su país, con el poder de elegir el modelo de ciudadanía que mejor le parezca, siempre y cuando tenga acceso a su conocimiento.

De acuerdo con la Declaración de los Derechos del Hombre y del Ciudadano de 1789, de la República Francesa, los artículos 11 y 14 determinan que la libre expresión de la opinión es uno de los derechos más valiosos del ser humano, así como el derecho del ciudadano para determinar la necesidad de su contribución pública (Consejo Constitucional de la República Francesa, 1789, pp. 2-3). Motivo por el que el tipo de ciudadanía -liberal, neoliberal, comunitarista o cualquier otra- no debería ser resultado de la imposición de ninguna institución nacional o extranjera, sino elegida en consciente libertad y autonomía, por el propio sujeto.

En adquisición de las teorías de autores e instituciones hasta el momento, la presente investigación se pregunta si a diferencia de una formación universitaria pública, que provoque un modelo de ciudadanía elegido en autonomía, consciente y dirigido hacia el bien común por convicción del individuo, se tiene que: ¿Los estudiantes de la universidad pública en la ciudad de Monterrey Nuevo León, muestran una tendencia consciente al individualismo, propio de la formación del modelo de ciudadanía neoliberal?. La vía analítica propuesta fue cuantitativa, dada la necesidad de medir variables tangibles.

Con el resultado del presente estudio se pretendió indagar si actualmente se provoca en la formación académica universitaria pública, un modelo de ciudadanía individualista que privilegia el beneficio personal antes o a costa del bien común (egoísta), sin que este sea elegido de manera libre y consciente por el alumno. 0 , por el contrario, se cumple el modelo "clásico" de formación universitaria pública, de preparar ciudadanos con la consciente convicción de preservar los valores humanos que procuran el bien común por encima de sus intereses personales. 
Es oportuno puntualizar que el perfil de ciudadanía (clásico) indicado en este modelo dirigido hacia la formación del interés comunitario y los valores humanos, no se refiere al concepto de ciudadanía cívica, porque aunque proviene del enfoque de la filosofía política en que se comprenden las relaciones que debe haber entre individuo y Estado, se dirige esencialmente a las virtudes cívicas del individuo (Olvera, 2008, pp. 1736), lo cual queda reducido en alcance para lo que busca el perfil de esta investigación. Tampoco se refiere al perfil de ciudadanía conservadora, que surge de la reacción político-intelectual-moral producto de la Revolución Francesa, porque el conservadurismo legitima un esquema de poder dominante que se requiere para el equilibrio del sistema, aunque para ello se necesite admitir desigualdad social y porque defiende una sola óptica del mundo, dado que teme a los cambios (Antón, 2012, pp. 14-16), lo cual inhibe el requerimiento de libertad de acción en comunidad. También se descarta el modelo del perfil de ciudadanía neoconservadora, porque su visión incluye el excepcionalismo al autodenominarse como la portadora del modelo de valores que debe ser emulado sin cuestionamiento, lo que la convierte en doctrina unilateral, nuevamente coartando la libertad democrática (Iglesias, 2015, pp. 91-92).

El modelo seguido para esta investigación es el de ciudadanía humanística, inspirado en el modelo que debe su origen al adoctrinamiento educativo helénico griego, en el que se buscaba -y se busca en esta investigación- el equilibrio en las aspiraciones humanas integrales con carácter gregario, mediante el concepto de que toda persona requiere de los demás en sociedad, con el fin de superar el interés individual que se basa primordialmente en lo técnico-económico (García, 1961 pp. 176, 178-179). Este modelo privilegia el sentido de comunidad y valores humanos, alejado del egoísmo y de la mayor parte de los conceptos asociados a una determinada política; cercana a la conceptualización de Warner Jaeger (Citado en García, 1961), como la visión filosófica del ser así como los valores del espíritu, virtudes reconocidas de lo que debe ser considerado como universidad (García, 1961, p. 179).

\section{Marco Teórico}

A diferencia de la instrucción puramente académica y el fin económico perseguido de influencia neoliberal, las instituciones de educación pública en 
las que se incluye a la universidad como educación superior, se consideran lugares con la misión de formar personas libres con la capacidad de producir conocimiento a partir del pensamiento reflexivo, así como difundirlo de manera masiva en cumplimiento del interés de la sociedad a la que sirven, dirigida a la atención de sus problemas, bienestar y equidad (Ornelas, 2009, pp. 96, 116).

Desde la época renacentista del siglo XV, el humanismo ha puntualizado el énfasis en los valores humanos; los humanistas como maestros de ese entonces en las universidades, se desempeñaban también en cargos públicos como cancilleres, dada su preparación para redactar documentos y acciones acordes con sus cargos políticos de manera profesional (Rivero, 2013, p. 85). Mientras los maestros tradicionales utilizaban predominantemente la lógica para sus explicaciones, los humanistas proclamaban la argumentación y la lectura como elementos fundamentales que servirían a sus estudiantes para comunicarse de forma más eficiente, dadas sus posibilidades de crítica textual y discursiva, lo cual podía servir además como medio al desarrollo de la identidad comunitaria (Rivero, 2013, pp. 86 y 87).

A pesar de la crítica a la formación humanística contra la científica, en relación de que la primera ostenta una fuerte tendencia al estudio ontológico de los autores en lugar de la experimentación, su exploración epistemológica se dirigió hacia la naturaleza, provocando el auge de la formación humanística universitaria que apareció en el siglo XIX en las instituciones públicas, donde se presentó un notable conocimiento de los clásicos que después se fue extinguiendo en el interés público al transcurrir el siglo XX, dado: el avance científico, las formas monótonas del aprendizaje y la especialización de los maestros -provocando que los alumnos no comprendieran sus lecciones-, por lo que se cuestionó al humanismo y su existencia (Rivero, 2013, pp. 83, 88 y 89).

Aunque las nuevas adquisiciones del conocimiento científico, así como la necesidad de los jóvenes estudiantes universitarios para insertarse al mercado laboral son importantes para el ser humano, la formación en humanidades es igualmente importante para su concientización como ciudadanos responsables, comprometidos con el bien común de la sociedad (Rivero, 2013, pp. 83, 92 y 93). Al respecto Pérez y Castaño (2016), 
advierten que la adecuada instrucción universitaria incluye la enseñanza epistemológica de una formación humana y crítica, que incentive la síntesis en la construcción del conocimiento para adaptarse a las nuevas situaciones tanto laborales como sociales, lo que requiere seguir vinculando a la filosofía con la ciencia, dada la crisis de tener actualmente demasiada información pero menor capacidad de síntesis interpretativa; es preciso que el estudiante domine un estado consciente y reflexivo de los datos para comprenderlos (Pérez y Castaño, 2016, pp. 192, 194, 195 y 196).

El estatus reflexivo en el conocimiento del ser humano es de utilidad para su emancipación personal, puesto que guía al individuo hacia un ser racional y autónomo, que pretende sujetarse a su propia identidad consciente (Miñana y Rodríguez, 2002, pp. 3-4, 14), aspecto que atañe especialmente a la educación superior, donde se forman los intelectuales y científicos que constituyen en gran parte el segmento crítico del pensamiento en la sociedad (Ornelas, 2009, pp. 84-85), pero a partir de 1960 con el Estado desarrollista, se redirigió al sistema educativo desde la lógica económica (Queiroz, 2001, pp. 92-96).

Aunque seguir su propio interés y por lo tanto su beneficio, se considere un camino racional positivo para que el ser humano pueda lograr su emancipación, lo cuestionable sería el propósito implícito en la subjetivación de esos intereses, pues como lo destacó Jürgen Habermas (1999), el actuar del sujeto soportado en la subjetividad de las necesidades de sus propios intereses en lugar de su actuar orientado hacia los valores, puede ser el problema de la represión de los intereses sociales (Habermas, 1999, pp. 190). De acuerdo a ese concepto, Hegel (citado en Follari, 2009) proponía al círculo familiar del sujeto, así como su relación con el Estado, como medios para la superación del subjetivismo personal, al superponer el interés universal antes que sus propios intereses, de tal manera que lo ético entre el individuo y la sociedad, sería la fórmula para lograr la internalización del vínculo social (Follari, 2009, pp. 169 y 178).

Aunque en el capitalismo el resto de los sujetos representa ser solo un objeto para el individuo, las normas de acción individual en la actualidad representan en la ideología económica una consecuencia para la colectividad y desde esta perspectiva, la toma de decisiones de manera racional representa una repercusión de importancia social pues afecta a los demás 
(Follari, 2009, pp. 169, 171 y 177). La universidad es una institución de fomento a la racionalización de conocimiento con sentido de nación (Landinelli, 2009, p. 206) y su responsabilidad se destaca en el argumento de la Organización de las Naciones Unidas para la Educación, la Ciencia y la Cultura [UNESCO] (1998), donde se declara que las instituciones de educación superior deberán someter sus acciones a la ética intelectual, mediante su libertad y autonomía, en respuesta a la formación educativa de ciudadanos conscientes para construir una sociedad reflexiva (UNESCO, 1998, p. 2).

Los argumentos destacan a la educación superior como un bien público, producto de la formación libre de intereses personales y dirigida al bien común (Fernández, 2009, p. 5), con una visión humanista como universidad pública, que destaca la formación de ser humano, en donde predomina la formación de la conciencia del individuo, el pensamiento crítico riguroso, la creatividad e imaginación (Jaramillo, 2012, pp. 197199); metas que se han desviado a consecuencia de la visión economicista, por lo que resulta imperioso recuperar la sensibilidad de solidaridad humana en la sociedad, la dignidad y la autonomía (Jaramillo, 2012, pp. $200,218-242)$.

Respecto a la personalización, Carl Rogers (citado en Artiles y otros, 1994) asegura que el ser humano pasa por un proceso de unificación entre el cuerpo físico que lo hace un animal como cualquier otro y sus necesidades humanas como son: sus afectos y un desarrollo que lo identifica social e individualmente, lo cual le confiere la toma de conciencia de su realidad (Artiles et al., 1994, p. 26), formación que en parte se origina en el período académico de la educación superior como ya se ha explicado.

La conciencia y percepción de solidaridad social son vocaciones adquiridas regularmente en la universidad pública, que ha utilizado medios como la autonomía y la libertad frente a los poderes civiles que históricamente han intentado su control (Tünnermann, 2008, pp. 19, 21-26 y 27-28), dicha resistencia tiene origen en la Edad Media, tiempo desde el cual la universidad ha buscado mantenerse independiente del poder del gobierno y la fe religiosa, aferrándose al objetivo de expresar y descubrir el conocimiento para la sociedad de manera desinteresada, 
admitiendo que su misión es mayor que la institución misma que representa (Bernasconi, 2014, pp. 2 y 3).

La labor de autonomía para llevar a cabo esa misión se ha complicado severamente para la universidad pública al enfrentar el cumplimiento paralelo a su objetivo académico-social, asociado simultáneamente al económico-global en dependencia del mandato de los recursos que recibe del Estado (Fernández, 2009, pp. 3-5), pues le representa objetivos contrarios.

Desde la teoría de la dependencia de los recursos, una sociedad solo puede ser autónoma en tanto pueda evitar ser impedida por otra para realizar su acción en libertad (Fernández, 2009, pp. 3-5). Para el filósofo Baruch Spinoza en su libro Tratado Político, cuando existe esta relación de impedimento, se presenta una alianza en la que al menos una de las partes experimenta miedo o se sujeta a la esperanza de obtener un beneficio de la otra parte, que solo podría recuperar -su autonomía- al romperse dicha alianza, perdiendo ese miedo o si el beneficio le fuera indiferente (Spinoza, 1986, pp. 108-109). Desde este juicio, a la universidad pública según su objetivo primordial, debe permitírsele soportar sus determinaciones únicamente en su debate interuniversitario de modo autosuficiente y en libertad moral, sin dependencia de mandatos ajenos al juicio de su propia razón, en atención del propósito de la ética ciudadana (Jaramillo, 2012, pp. 210-213 y 215-216).

Sintetizando la teoría adquirida de los autores consultados y las fuentes internacionales, es posible integrar las relaciones y dirección actual de la influencia formativa proveniente de la universidad pública en el estudiante de educación superior, como se presenta en la figura 1.

De acuerdo con los autores, el sujeto estudiante de universidad pública en general actualmente, se encuentra influenciado por el pragmatismo que le infiere la política educativa internacional con enfoque neoliberal, en el que primordialmente evalúa sus propias necesidades físicas de manera subjetiva, mediante la intención determinada de su posible posición laboral económica y por lo tanto el bienestar de su propio interés personal. En el caso de que se trate de un estudiante que intenta una interpretación de su realidad como sujeto humano social, ese alcance -según los autoresllegará únicamente a referir que la realidad sigue siendo pragmática para 
el resto de las personas, por lo que esto aunado a su -supuesta- escasa instrucción en materia de síntesis de la problemática humana, no le alcanzará para formar conciencia de su entorno de manera autónoma, motivo por el que regresará a enfocarse en su interés personal.

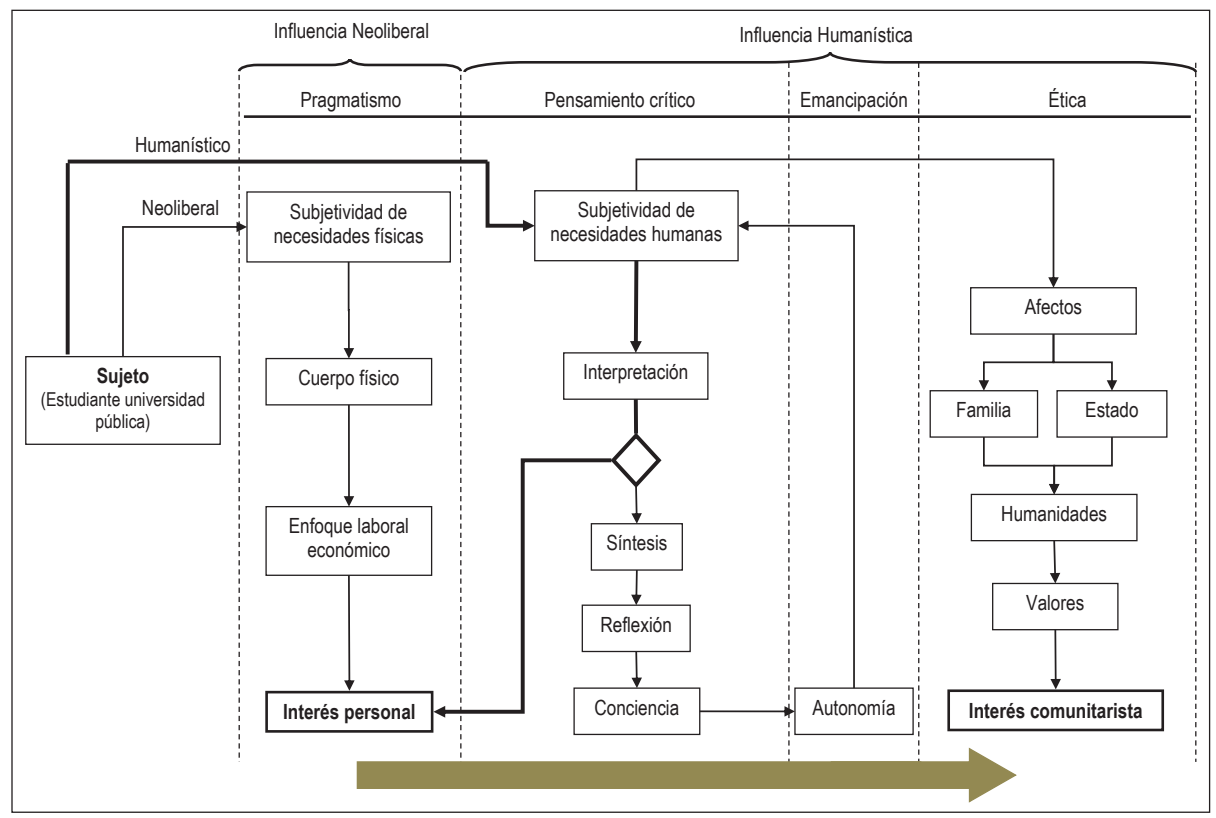

Figura 1. pp. Tipo de influencia formativa en universidad pública según autores

Fuente: Elaboración propia

Los autores refieren que el ámbito para llegar a una formación integral dirigida hacia el interés comunitarista como finalidad principal, está muy alejado de la formación para el sujeto actual, puesto que para alcanzar dicho fin, tendría que reforzarse su capacidad de síntesis enfocada a ser una persona con conciencia reflexiva en sus posibilidades de pensamiento crítico, para que desde sus juicios autónomos pueda tener una diferente subjetivación de las necesidades humanas, tanto propias como las de sus círculos cercanos y sociales en general, de tal manera que se encuentre preparado para sensibilizar los valores humanos que le permitan dirigir su interés en la comunidad y en el Estado, del que ha recibido estudios superiores. 


\section{Metodología}

Esta investigación se interesó en descubrir si existe una tendencia consciente de educación superior individualista en los estudiantes de la universidad pública, en la ciudad de Monterrey Nuevo León, México, que dirige a objetivos pragmáticos personales del sujeto en lugar del bien comunitario. Para comprobarlo, se estudió dicho efecto en sujetos que son (y fueron) estudiantes universitarios en instituciones de educación pública. La pregunta de investigación fue: ¿Los estudiantes de la universidad pública en la ciudad de Monterrey Nuevo León, muestran una tendencia consciente al individualismo, propio de la formación del modelo de ciudadanía neoliberal?

El concepto de individuo tiene una significación intrínseca desconocida, dado que parte de su propia indefinición; y la noción de persona, se entiende como caracterización o personaje con un rol especifico, conocido a partir de su representación a la que debe su existencia, por lo que el individuo solo puede ser conocido a partir de su personalidad (Valverde, 2014, pp. 29-31). Dado que el individuo liberal es motivado con base en la utilidad que puede generar el valor de las cosas, y esta condición es conducida únicamente por los impulsos generados por su cuerpo, es un ente egoísta (Valverde, 2014, pp. 29 y 32).

A partir de este enfoque egoísta del ser humano que busca su propio bienestar y que al intentar su ganancia económica individual requiere del recurso humano como objeto de compra, que le devengue esa posibilidad de explotación dentro de la expresión de la ética del trabajo del capitalismo occidental, el neoliberalismo resulta ser un mecanismo que observa a los seres humanos como mercancías sin dignidad y por lo tanto sin sentido para un humanista (Monares, 2005, pp. 1-4), de tal manera que el sistema capitalista liberal, requiere de individuos en su forma "cosificada ${ }^{3}$ ", sin dominio consciente de su propia persona para lograr su manipulación ética y moral mediante el consumismo (Valverde, 2014, pp. 34 у 39).

Con motivo de identificar la tendencia (neoliberal o humanística) de los sujetos participantes en el estudio, así como la evaluación de su consistencia en dicha convicción, se construyó un instrumento de recolección con reactivos para la medición en cuatro variables que fueron: 1) Perfil -neoliberal o humanístico-, 2) Conciencia -pragmática o colectiva-, 3) Valores -personales o sociales- e 4) Interés -personal o comunitario-. 
El proceso para este diseño se fundamentó en medir cada una de las variables por separado, para luego promediar del número dos a la cuatro, y finalmente analizar si había congruencia entre la opinión de esas cuatro variables -concentrándola en una sola, denominada como: "5) Interés sin Perfil"-, respecto a la variable 1 (Perfil) que previamente calificaba a cada sujeto. Esto último para acreditar que los sujetos hacían sus juicios de manera consciente y congruente a su perfil, o por el contrario, había contradicciones. Se utilizó una escala que incluía 4 niveles de calificación en lugar de medición dicotómica para las variables, porque el objetivo era buscar la tendencia en los perfiles presentados y la escala dicotómica presentaba una determinación tajante del perfil en lugar de la tendencia.

Cada pregunta tenía cuatro opciones para su respuesta para evitar una central, que invalidara la tendencia hacia uno de los dos perfiles; la escala de medición fue de 1 a 10, que acreditaría las dos posibles respuestas de menor calificación (2,5 y 5) como Perfil Neoliberal, y las otras dos restantes de mayor calificación (7,5 y 10) como Perfil Humanístico, por lo que se consideró una calificación igual o menor a 6,25 como el promedio obtenido del resultado total, que clasificaría al sujeto participante como Perfil Neoliberal y mayor a esta (hasta 10), como Perfil Humanístico. De igual modo operó para el resto de las variables mencionadas. Dada la importancia de la secuencia en las preguntas y su puntaje, estas se presentaron al sujeto encuestado de manera no secuencial, para evitar el posible sesgo por manipulación de las respuestas.

Para poder responder a la presente investigación, fue necesaria la comparación del resultado del grupo de estudio, con otro de similares características pero que no estuviera expuesto al estímulo del modelo actual globalizado mediante la influencia institucional neoliberal que proponen los autores consultados, por lo que se utilizaron dos muestras de sujetos; una de estudiantes de universidad pública actual activos y otra de profesionistas ex/estudiantes de universidad pública, producto del modelo anterior al neoliberal. Precisado este criterio, la pregunta planteada se convirtió en la hipótesis para esta investigación, como sigue:

$\mathrm{H}_{1}$ - Los estudiantes de la universidad pública en la ciudad de Monterrey Nuevo León, muestran una tendencia consciente al individualismo, propio de la formación del modelo de ciudadanía neoliberal. 
La hipótesis se contestaría al exponerla en contraste con el comportamiento encontrado en la muestra $2\left(G_{2}\right)$. El experimento constó de 379 sujetos participantes en dos muestras, sin utilizar grupo de control; la muestra $1\left(G_{1}\right)$ compuesta por 197 hombres y mujeres, estudiantes actualmente activos de su último año en la carrera de negocios internacionales en la Facultad de Contaduría Pública y Administración, de la Universidad Autónoma de Nuevo León (una universidad pública con una población aproximada de 14.500 estudiantes de nivel licenciatura, ubicada al norte de la República Mexicana), con el objetivo de obtener su representatividad como sujetos expuestos al modelo actual de educación universitaria pública; y la muestra $2\left(\mathrm{G}_{2}\right)$ compuesta por 182 hombres y mujeres profesionistas mayores de 60 años de edad, que fueron estudiantes de universidad pública aproximadamente en los ochentas, pues se buscó obtener de estos sujetos la representatividad del anterior modelo (clásico) al formativo neoliberal, situado en el tiempo que proponen los autores (Bernasconi, 2014; Miñana y Rodríguez, 2002; Salazar, 2018; Ornelas, 2009; Queiroz, 2001). El diseño experimental fue el siguiente:

$$
\begin{aligned}
& G_{1} X_{1} 0_{1} \\
& G_{2} X_{1} 0_{2}
\end{aligned}
$$

Se aplicó el instrumento de recolección en forma de encuesta $\left(\mathrm{X}_{1}\right)$ a la muestra $1\left(G_{1}\right)$, realizándose una primera evaluación y obteniéndose su resultado $\left(0_{1}\right)$, posteriormente se utilizó el muestreo por bola de nieve, para recopilar a los sujetos participantes de la muestra $2\left(G_{2}\right)$, solicitándoles un candidato a cada uno de los sujetos participantes de la muestra $1\left(G_{1}\right)$, con el objetivo de recopilar primordialmente a sus círculos sociales cercanos como candidatos para dicha muestra $\left(G_{2}\right)$. El intento de estos "lazos cercanos", fue para dar seguimiento a las teorías de la subjetividad de intereses personales o valores, así como de la relación de su círculo familiar y la que tienen con el Estado, de Habermas (1999) y Hegel (citado en Follari, 2009) respectivamente. De esta recopilación y aplicando el mismo instrumento $\left(X_{1}\right)$, se obtuvo el cálculo para la segunda evaluación y resultados $\left(0_{2}\right)$ de la muestra 2.

La investigación fue cuantitativa, dada la comprobación de la hipótesis mediante algoritmos matemáticos y pruebas estadísticas, y no probabilística dado que la muestra 1 fue recopilada mediante la técnica de 
muestreo por juicio de investigador y la muestra 2, como ya se comentó, mediante la técnica de bola de nieve.

Dado que la finalidad de la investigación consistía en encontrar el perfil de ciudadanía que identificaría al sujeto participante en ambas muestras, se evitó revelar este motivo original a los sujetos y en su lugar se les indicó que se trataba de una investigación sobre experiencia universitaria y pobreza, para lograr que el esfuerzo en sus respuestas se enfocara en reflejar su opinión de lo que fue (y está siendo) su paso por el grado universitario, así como su verdadera vocación hacia lo que significa la carencia del recurso económico propio y ajeno en el instrumento de recolección.

\section{Resultados}

En función de que la variable 5 (Interés sin Perfil) resultaría la concentradora de las variables independientes en la investigación, se realizó para ella la prueba de Kolmogorov-Smirnov en ambas muestras, presentando normalidad en sus datos con resultado de $p=0,141$, para la muestra 1 y de $p=0,327$ para la muestra 2, por lo que se procedió a efectuar una prueba paramétrica para contestar la hipótesis de la investigación, intentando comprobar que la media de la calificación obtenida de los profesionistas mayores de 60 años de edad presentaría una mayor tendencia general al Perfil Humanístico, siendo mayor a la media de los estudiantes de universidad pública actuales, esperando que resultara con mayor tendencia al Perfil Neoliberal, utilizando un nivel de significancia de $\alpha=0,05$, mediante la fórmula para la comparación de medias (Z) con el siguiente diseño de hipótesis estadística:

$$
\begin{aligned}
& H_{o}=\mu_{2} \leq \mu_{1} \\
& H_{1}=\mu_{2}>\mu_{1}
\end{aligned}
$$

Donde:

$\mu_{1}=$ Sujetos estudiantes de universidad pública actuales

$\mu_{2}=$ Sujetos profesionistas mayores de 60 años, c/estudios en universidad pública 
El resultado del experimento fue de $\mathrm{Z}=-0,862$ calculada $<\mathrm{Z}_{\alpha}=1,645$; por lo que no se rechazó $H_{0}$. Debido a ello, no hubo evidencia suficiente para suponer que la media de la calificación obtenida de los profesionistas de universidad pública mayores de 60 años de edad presentaba una mayor tendencia general al Perfil Humanístico, con lo que no fue posible determinar que los profesionistas de universidad pública mayores de 60 años de edad presentaban mayor tendencia general al Perfil Humanístico que los estudiantes de universidad pública actuales.

Al revisar los registros que designaban el resultado de la variable 1 a cada sujeto de las muestras con su perfil respectivo, se encontró que de los 197 participantes en la muestra 1 (estudiantes) el 64\% resultó con perfil neoliberal, mientras que de los 182 en la muestra 2 (profesionistas) solo el 51\% (prácticamente la mitad) fueron determinados con ese perfil, lo cual indicó una diferencia significativa que marcó una tendencia de mayor perfil neoliberal en número de los sujetos estudiantes de universidad pública actual que en los profesionistas. Motivo por el que se decidió realizar una nueva comprobación de hipótesis, pero en esta ocasión dos pruebas; una para cada subgrupo utilizando nuevamente a la variable 5 (Interés sin Perfil): una prueba para los sujetos resultantes con perfil neoliberal y otra para los de perfil humanístico. De esta manera se buscó interpretar que no habiéndose encontrado una tendencia general de la muestra de estudiantes de universidad pública actuales al perfil neoliberal, habría con esta segunda prueba la posibilidad de encontrar una tendencia sólida dentro de cada perfil en dichos sujetos, así como en los profesionistas, de tal manera que esto identificara la existencia de una formación particularizada a quien fuera susceptible al perfil neoliberal, no en lo general.

Se procedió primeramente a realizar la prueba de normalidad para ambos pares de muestras, pero a pesar de que en todos los casos (4 muestras) se presentó un resultado positivo para la prueba de KolmogorovSmirnov (Estudiantes Neoliberal: $p=0,457$; Profesionistas Neoliberal: $p=$ 0,281; Estudiantes Humanístico: $p=0$,293; Profesionistas Humanístico: $p$ $=0,502$ ), se decidió aplicar la prueba no paramétrica de $X^{2}$ para contar con una tabla de contingencia que permitiera observar las diferencias entre los grupos del análisis. 
El detalle del razonamiento fue que del grupo de participantes de la muestra 1, efectivamente 127 fueron clasificados con perfil neoliberal, pero según sus respuestas, 65 de ellos refrendaron su perfil dentro del Rango Neoliberal y los restantes 62 calificaron dentro del Rango Humanístico. Del mismo modo, 92 profesionistas de universidad pública mayores de 60 años clasificaron con perfil neoliberal, resultando 39 de ellos con refrendo en dicho Rango Neoliberal y los 53 restantes dentro del Rango Humanístico. Se buscó comprobar que la media en ambos rangos de los estudiantes de universidad pública actuales presentaba una diferencia al perfil neoliberal comparada con la media de los profesionistas mayores de 60 años de edad, utilizando un nivel de confianza del 95\%, mediante la siguiente hipótesis estadística para las dos pruebas (neoliberales y humanísticos):

$$
\begin{aligned}
& H_{o}=\mu_{2}=\mu_{1} \\
& H_{1}=\mu_{2} \neq \mu_{1}
\end{aligned}
$$

Donde:

$\mu_{1}=$ Sujetos estudiantes de universidad pública actuales

$\mu_{2}=$ Sujetos profesionistas mayores de 60 años, c/estudios en universidad pública

El resultado del experimento para el perfil neoliberal de los sujetos en las dos muestras fue de $X^{2}$ calculada $(1,653)<X^{2}$ de la tabla $(3,84)$; por lo que no se rechazó $\mathrm{H}_{0}$. Nuevamente debido a que no se rechazó $\mathrm{H}_{\mathrm{o}}$, se concluyó que no hubo evidencia suficiente para aceptar que la media de la calificación obtenida de ambos rangos (neoliberales y humanísticos) de los estudiantes de universidad pública actuales clasificados con perfil neoliberal, era diferente en comparación con la media de los mismos rangos para los profesionistas de universidad pública mayores de 60 años.

De igual manera, el resultado del experimento para el perfil humanístico de los sujetos en las dos muestras fue de $X^{2}{ }_{\text {calculada }}(0,1206)<X^{2}$ de la tabla $(3,84)$; por lo que no se rechazó $H_{0}$. Nuevamente debido a que no se rechazó $\mathrm{H}_{\mathrm{o}}$, se concluyó que no hubo evidencia suficiente para aceptar que la media de la calificación obtenida de ambos rangos (neoliberales y humanísticos) de los estudiantes de universidad pública actuales clasificados con perfil 
humanístico, era diferente en comparación con la media de los mismos rangos para los profesionistas de universidad pública mayores de 60 años.

Las tablas de contingencia para ambas pruebas se presentan en las Tablas 1 y 2.

Tabla 1

Número de sujetos con Perfil Neoliberal

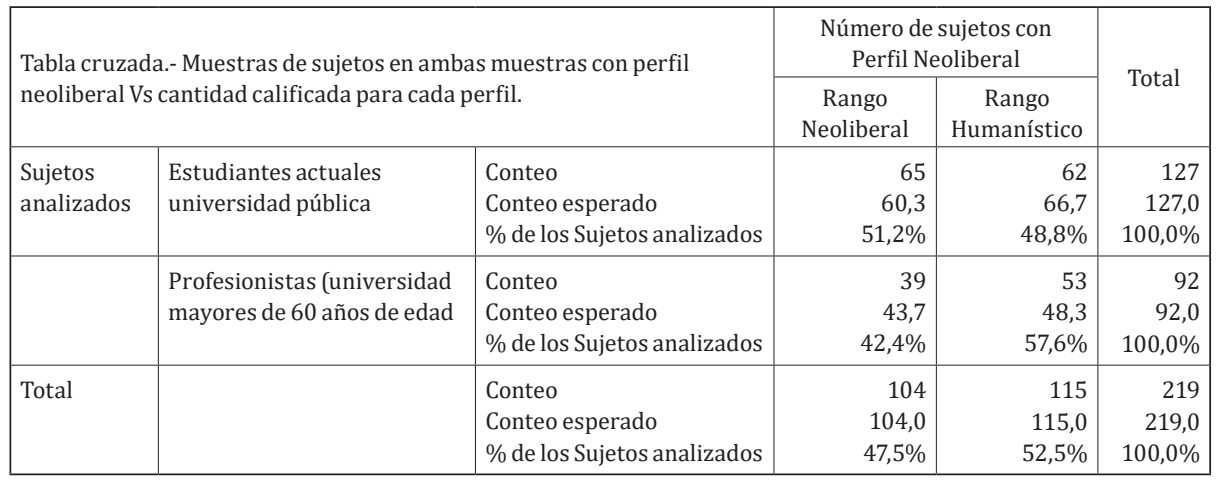

Fuente: elaboración propia

Tabla 2

Número de sujetos con Perfil Humanístico

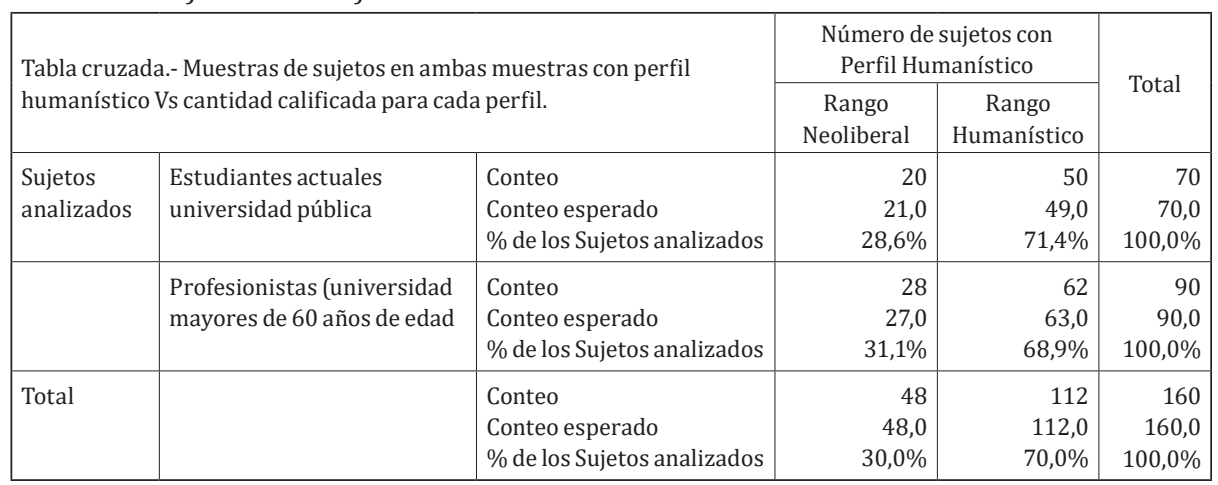

Fuente: elaboración propia

Mediante esta segunda prueba, pudo descartarse la posibilidad de tendencia al perfil neoliberal en sujetos susceptibles a dicho perfil en las dos muestras de sujetos participantes, pero la revisión visual de las tablas 
de contingencia de la prueba, demostraron que los sujetos que acreditaron como perfil neoliberal en la muestra de estudiantes, debieron presentar una mayoría en número dentro de la calificación del rango neoliberal frente al rango humanitarista, pero casi se presentó en la misma magnitud $(51,2 \%)$, mientras que los sujetos de la muestra de profesionistas fue aún menor $(42,4 \%)$, lo cual fue un indicativo de que ambos grupos de sujetos no tuvieron una posición sólida, congruente a sus convicciones en uno u otro perfil. La tabla de contingencia en cuanto al perfil humanístico demostró una mayor solidez en ambos rangos de perfil, siendo que el esperado en ambos casos tuvo una cantidad superior al promedio $(71,4 \%$ en los estudiantes y 68,9\% en los profesionistas).

Con la observación de esta segunda prueba, se decidió realizar una revisión de los promedios por perfil en cada muestra, pero en el resto de las variables independientes del estudio (sin concentrarlas en la variable 5), encontrándose que el grupo de 127 estudiantes de universidad pública que fue clasificado con perfil neoliberal, obtuvo una calificación de 7,48 en escala entre 1 y 10 en Valores Sociales (variable 3), mientras que el grupo de 92 profesionistas mayores de 60 años clasificado con el mismo perfil, obtuvo una calificación de 6,71 bajo la misma escala. Dado que la calificación igual o inferior a 6,25 en la escala, determina con precisión al perfil neoliberal, se sospecha que hay conflicto en la media que presenta la calificación para esta variable en la muestra 1 (estudiantes), por lo que se buscó comprobar que la media de calificación de los estudiantes de universidad pública actuales presentaba una menor congruencia al perfil neoliberal respecto a su opinión en Valores Sociales siendo diferente a la media que lo identifica, comparada con la media de los profesionistas mayores de 60 años de edad, utilizando un nivel de confianza del 95\%, mediante la siguiente hipótesis estadística:

$$
\begin{aligned}
& H_{o}=\mu_{2}=\mu_{1} \\
& H_{1}=\mu_{2} \neq \mu_{1}
\end{aligned}
$$

Donde:

$\mu_{1}=$ Sujetos estudiantes de universidad pública actuales

$\mu_{2}=$ Sujetos profesionistas mayores de 60 años, c/estudios en universidad pública 
El resultado del experimento en esta tercera prueba para el perfil neoliberal de los sujetos en las dos muestras fue de $X^{2}$ calculada $(12,819)>$ $X^{2}$ de la tabla $(7,81)$; por lo que se rechazó $H_{0}$. En esta ocasión, debido a que se rechazó $\mathrm{H}_{\mathrm{o}}$, se concluyó que hubo evidencia suficiente para aceptar que la media de la calificación obtenida de los estudiantes actuales de universidad pública, clasificados con perfil neoliberal respecto a su opinión en los Valores Sociales, es diferente en comparación con la media de los profesionistas de universidad pública mayores de 60 años.

La tabla de contingencia al respecto (Tabla 3) entrega evidencia fehaciente de este resultado, pues al realizar el conteo de los sujetos de cada muestra acreditados con perfil neoliberal, se destaca a la mayor parte de los participantes en la muestra de estudiantes $(0,8+52,8=53,6 \%)$, calificando alto en la escala de la variable 3 (Valores Sociales), mientras que, dado su perfil, lo congruente sería esperar un dato por debajo del 50\%. Lo cual si sucedió con la muestra de los profesionistas $(8,7+37=45,7 \%)$

Tabla 3

pp. Calificación de Valores Sociales (variable 3), Perfil Neoliberal.

\begin{tabular}{|c|c|c|c|c|c|c|c|}
\hline \multirow{2}{*}{\multicolumn{3}{|c|}{$\begin{array}{l}\text { Tabla cruzada.- Muestras de sujetos en ambas muestras con perfil } \\
\text { neoliberal Vs calificación puntual variable valores sociales. }\end{array}$}} & \multicolumn{4}{|c|}{ Calificación Valores Sociales } & \multirow{2}{*}{ Total } \\
\hline & & & 2,5 & 5 & 7,5 & 10 & \\
\hline \multirow{6}{*}{$\begin{array}{l}\text { Sujetos } \\
\text { analizados }\end{array}$} & \multirow{3}{*}{$\begin{array}{l}\text { Estudiantes actuales } \\
\text { universidad pública }\end{array}$} & \multirow{3}{*}{\begin{tabular}{|l|} 
Conteo \\
Conteo esperado \\
$\%$ de los Sujetos analizados
\end{tabular}} & 9 & 50 & 1 & 67 & 127 \\
\hline & & & 12,8 & 50,5 & 5,2 & 58,6 & 127,0 \\
\hline & & & $7,1 \%$ & $39,4 \%$ & $0,8 \%$ & $52,8 \%$ & $100,0 \%$ \\
\hline & \multirow{3}{*}{$\begin{array}{l}\text { Profesionistas (universidad } \\
\text { mayores de } 60 \text { años de edad }\end{array}$} & Conteo & 13 & 37 & 8 & 34 & 92 \\
\hline & & Conteo esperado & 9,2 & 36,5 & 3,8 & 42,4 & 92,0 \\
\hline & & $\%$ de los Sujetos analizados & $14,1 \%$ & $40,2 \%$ & $8,7 \%$ & $37,0 \%$ & $100,0 \%$ \\
\hline \multirow[t]{3}{*}{ Total } & & Conteo & 22 & 87 & 9 & 101 & 219 \\
\hline & & Conteo esperado & 22,0 & 87,0 & 9,0 & 101,0 & 219,0 \\
\hline & & \% de los Sujetos analizados & $10,0 \%$ & $39,7 \%$ & $4,1 \%$ & $46,1 \%$ & $100,0 \%$ \\
\hline
\end{tabular}

Fuente: elaboración propia

Con base en la misma apreciación, pero en la variable 4, se encontró que los 70 estudiantes de universidad pública actuales que fueron clasificados con perfil humanístico obtuvieron una calificación de 4,86 en escala entre 1 y 10 en Interés Comunitario, mientras que el grupo de 90 profesionistas mayores de 60 años clasificado con el mismo perfil, obtuvo una calificación de 6,39 bajo la misma escala. Nuevamente y con base en que la calificación superior a 6,25 en la escala determina con precisión al perfil humanístico, se pensó en la presencia del mismo conflicto en la 
media de calificación de los sujetos de la muestra 1 (estudiantes), por lo que nuevamente se buscó comprobar que la media de calificación de los estudiantes de universidad pública actuales, presentaba una menor congruencia al perfil humanístico respecto a su opinión en Interés Comunitario siendo diferente a la media que lo identificaba, comparada con la media de los profesionistas mayores de 60 años de edad, utilizando un nivel de confianza del 95\%, mediante la misma hipótesis estadística para la prueba de $X^{2}$.

$$
\begin{aligned}
& H_{o}=\mu_{2}=\mu_{1} \\
& H_{1}=\mu_{2} \neq \mu_{1}
\end{aligned}
$$

Donde:

$\mu_{1}=$ Sujetos estudiantes de universidad pública actuales

$\mu_{2}=$ Sujetos profesionistas mayores de 60 años, c/estudios en universidad pública

El resultado del experimento para el perfil humanístico de los sujetos en las dos muestras fue de $X^{2}$ calculada $(13,579)>X^{2}$ de la tabla $(7,81)$; por lo que se rechazó $\mathrm{H}_{\mathrm{o}}$. Debido a que se rechazó $\mathrm{H}_{\mathrm{o}}$, se concluyó que hubo evidencia suficiente para aceptar que la media de la calificación obtenida de los estudiantes actuales de universidad pública, clasificados con perfil humanístico respecto a su opinión en el Interés Comunitario, es diferente en comparación con la media de los profesionistas de universidad pública mayores de 60 años.

Tabla 4

\begin{tabular}{|c|c|c|c|c|c|c|c|}
\hline \multirow{2}{*}{\multicolumn{3}{|c|}{$\begin{array}{l}\text { Tabla cruzada.- Muestras de sujetos en ambas muestras con perfil } \\
\text { humanístico Vs calificación puntual variable interés comunitario. }\end{array}$}} & \multicolumn{4}{|c|}{ Calificación Interés Comunitario } & \multirow{2}{*}{ Total } \\
\hline & & & 2,5 & 5 & 7,5 & 10 & \\
\hline \multirow{6}{*}{$\begin{array}{l}\text { Sujetos } \\
\text { analizados }\end{array}$} & \multirow{3}{*}{$\begin{array}{l}\text { Estudiantes actuales } \\
\text { universidad pública }\end{array}$} & \multirow{3}{*}{\begin{tabular}{|l|} 
Conteo \\
Conteo esperado \\
$\%$ de los Sujetos analizados
\end{tabular}} & 34 & 15 & 12 & 9 & 70 \\
\hline & & & 24,5 & 13,6 & 19,3 & 12,7 & 70,0 \\
\hline & & & $48,6 \%$ & $21,4 \%$ & $17,1 \%$ & $12,9 \%$ & $100,0 \%$ \\
\hline & \multirow{3}{*}{$\begin{array}{l}\text { Profesionistas (universidad } \\
\text { mayores de } 60 \text { años de edad }\end{array}$} & Conteo & 22 & 16 & 32 & 20 & 90 \\
\hline & & Conteo esperado & 31,5 & 17,4 & 24,8 & 16,3 & 90,0 \\
\hline & & $\%$ de los Sujetos analizados & $24,4 \%$ & $17,8 \%$ & $35,6 \%$ & $22,2 \%$ & $100,0 \%$ \\
\hline \multirow[t]{3}{*}{ Total } & & Conteo & 56 & 31 & 44 & 29 & 160 \\
\hline & & Conteo esperado & 56,0 & 31,0 & 44,0 & 29,0 & 160,0 \\
\hline & & $\%$ de los Sujetos analizados & $35,0 \%$ & $19,4 \%$ & $27,5 \%$ & $18,1 \%$ & $100,0 \%$ \\
\hline
\end{tabular}

pp. Calificación de Interés Comunitario (variable 4), Perfil Humanístico.

Fuente, pp. elaboración propia 
En esta ocasión la tabla de contingencia correspondiente al análisis de la variable 4 (Interés Comunitario) (Tabla 4) entregó evidencia de mayor contundencia que la anterior, pues al realizar el conteo de los sujetos de cada muestra acreditados con perfil humanístico, se destacó a la mayor parte de los participantes en la muestra de estudiantes, calificando extremadamente por debajo del promedio en cuanto a su Interés Comunitario $(17,1+12,9=30 \%)$, mientras que en congruencia a su perfil, debió de presentarse un dato superior al 50\%. Lo cual si sucedió con la muestra de los profesionistas $(35,6+22,2=57,8 \%)$, que de hecho dejó en claro su tendencia al interés en la comunidad.

\section{Discusión}

Se utilizó una prueba de contraste mediante dos muestras de sujetos, representando cada una la vivencia de su tiempo como universitario de institución pública para contestar la hipótesis planteada en la presente investigación. La primera prueba, se realizó con base en la idea homogénea del perfil del sujeto que integraba cada uno de los grupos, asumiendo que al formar parte de la muestra de universitarios de la década de los ochentas (profesionistas), el comportamiento y perfil de cada sujeto sería igual; de la misma manera se asumió en la muestra de estudiantes actuales.

El resultado fue que no había una tendencia general en los profesionistas de universidad pública mayores de 60 años hacia el perfil humanístico, en comparación con los estudiantes actuales. Al respecto, la investigación de Álvarez (2014), compuesta por ciudadanos Costarricenses participantes en: partidos políticos; asociaciones de desarrollo municipal y otros que son militantes de movimientos sociales sin tener adscripción en partidos políticos, efectuada mediante 6 grupos de enfoque, demostró que a 30 años de la implementación gradual de reformas neoliberales en el país, los ciudadanos no eran capaces de precisar los cambios macroeconómicos y sus implicaciones en la democracia de su país, así como en el bienestar humano (Álvarez, 2014, pp. 4-37), a pesar de que los sujetos lograron identificar efectos negativos en su bienestar relativos a dichas reformas, no pudieron especificar los detalles de incidencia producto de la implementación (Álvarez, 2014, p. 50). 
Aunque se refiere a otro país (diferente a México) pero Latinoamericano, dicha investigación entregó evidencia de la posible desinformación del ciudadano Costarricense sobre las precisiones del neoliberalismo en su país, pero no de su desinterés, puesto que pudieron asociar las medidas con efectos negativos en su bienestar, esto puede ser un indicativo de que en el experimento realizado en México está sucediendo un efecto similar de desinformación que provoca una distorsión en la opinión general inter-generacional. Por ello y debido a que en la presente investigación se encontró una diferencia porcentual significativa de individuos con perfil neoliberal en la muestra de estudiantes (64\%), en comparación con la de profesionistas (51\%) que identificó una mayor tendencia en la cantidad de sujetos neoliberales en la muestra de estudiantes actuales, se procedió a la aplicación de una segunda prueba de hipótesis que igualmente determinó que no había diferencia en los rangos de calificación como neoliberal o humanístico en ambas muestras, lo que descartó la existencia de una formación particularizada que hiciera susceptible a los sujetos de perfil neoliberal hacia dicho comportamiento. La prueba se extendió en las muestras del perfil humanístico con los mismos resultados, aunque en este último caso demostrando consistencia, lo que provocó una confusión en su lectura.

Ante este resultado, pudo distinguirse que posiblemente habría un tipo de generación que pasa por auto-representación o confusión en su comportamiento. Para Lash (citado en Fair, 2008), existió: una etapa de desarrollo tradicional de la sociedad, que se basaba en los lazos comunitarios entre los individuos; otra que denominó como modernidad simple, con fundamento en lazos colectivos; y actualmente se presenta la que denomina como modernidad reflexiva, que se caracteriza por la autonomía de los actores y las estructuras sociales como los cambios en el Estado asistencial (Fair, 2008, p. 12), las últimas dos etapas ocurrieron en la transición marcada desde el fin de la producción industrial en el modernismo, hasta el inicio de la era del conocimiento técnico y la información en el posmodernismo (Fair, 2008, p. 2), estas etapas causaron modificaciones en el comportamiento social producto de la transición, provocando una desorientación en los investigadores que intentan una lectura en la comprensión sistemática de la organización social, ya que el surgimiento de diversas formas de fragmentación social por intereses heterogéneos, fomentaron el pluralismo y nuevos formatos de ciudadanía auto-representada y despolitizada (Fair, 2008, p. 2). 
Detalles particulares detectados en las tablas de contingencia de la prueba (Tablas 2 y 3) demostraron la evidencia de esta contradicción. Hidalgo (2017), realizó una investigación por medio de 48 entrevistas a estudiantes de licenciatura de las carreras de: ciencias económico-administrativas, ciencias exactas e ingenierías, así como ciencias sociales y humanidades, en la Universidad de Guadalajara en México (una universidad pública ubicada en la entidad federativa que ocupa el tercer lugar con mayor población estudiantil en el país), con el fin de encontrar las formas de representación social en los estudiantes que les servían para relacionarse con los otros y su entorno (Hidalgo, 2017, pp. 175-181).

Los resultados de Hidalgo (2017), determinaron que las expectativas de los jóvenes se enfocaban en su proyecto individual tanto como en el colectivo, argumentando para este último (alcance en lo colectivo) que se asumían como elementos del cambio para la mejora en las condiciones de la sociedad como su interés prioritario, y como segundo punto (en lo individual) declararon que aunque las oportunidades de empleo existían, admitieron ser explotados por sus empleadores; estas características no aparecieron de manera general en los entrevistados, sino que fueron argumentadas conforme a la experiencia propia en función estrecha de su adscripción a grupos diferenciados (Hidalgo, 2017, pp. 183-198). En seguimiento a estas características homologadas a la evidencia de la presente investigación, se procedió a la realización de la tercera (última) prueba de hipótesis pero para el resto de las variables independientes, encontrando diferencias significativas comprobables y contradictorias, en la opinión de los sujetos de la muestra de estudiantes con respecto a sus valores sociales (perfil neoliberal) e interés comunitario (perfil humanitarista), lo cual no ocurrió con la muestra de los profesionistas que presentaron congruencia en sus perfiles respecto a las mismas variables.

\section{Reflexiones finales}

Mediante las pruebas estadísticas aplicadas a los sujetos participantes en las muestras de estudio, se encontró que los profesionistas de universidad pública mayores de 60 años no presentaron una mayor tendencia general al humanismo comparada con los estudiantes de universidad pública actuales, asimismo ambas muestras generales, mostraron medias 
similares en rango de opinión general tanto hacia el perfil neoliberal como al humanístico.

Por lo que, en respuesta a la hipótesis propuesta, la presente investigación determinó que, en los estudiantes de la universidad pública en la ciudad de Monterrey Nuevo León, México, no existe una tendencia general consciente al individualismo, propio de la formación del modelo de ciudadanía neoliberal. Pero se encontró una tendencia mayor en número de estudiantes de universidad pública actual (64\%) hacia el perfil neoliberal que en los profesionistas (51\%). Adicionalmente los estudiantes de universidad pública actuales clasificados con perfil neoliberal, mostraron una media diferente respecto a su opinión en los valores sociales; los clasificados con perfil humanístico, mostraron una media diferente respecto a su opinión en el interés comunitario, ambos en comparación con la de los profesionistas de universidad pública mayores de 60 años de edad, lo que determinó incongruencia en la opinión de estudiantes de universidad pública actuales, correspondiente a su propio perfil.

Estos hallazgos sirvieron a la presente investigación para concluir que existe una tendencia particularizada tendiente al neoliberalismo en los estudiantes de la universidad pública en la ciudad de Monterrey Nuevo León, México, que al resultar inconsistente, pudiera provenir de la desinformación al respecto de lo que significa el pensamiento neoliberal; de una propensión al consumismo mediático o de cualquier otra razón, pero que de todas maneras le provoca confusión en su postura como perfil neoliberal o humanístico, al no presentar una opinión congruente con su comportamiento consciente. Teoría que corresponde a la incapacidad de síntesis de la sensibilidad humana en sociedad que requiere el estudiante universitario en la actualidad, propuesta por los autores consultados (Fernández, 2009, p. 5; Jaramillo, 2012, pp. 200, 218-242; Artiles et al., 1994, p. 26).

Algunos de los estudiantes entrevistados en la investigación de la Universidad de Guadalajara elaborada por Hidalgo (2017), argumentaron que sus expectativas estaban supeditadas a la aceptación social de su entorno inmediato, lo cual condicionó la carrera que eligieron porque estaba sujeta a la aprobación de sus familiares no a sus gustos personales, motivo por el cual experimentan exceso de dependencia, estrés y falta de liberad (Hidalgo, 2017, pp. 183-198). A razón de las conclusiones en la presente 
investigación, se recomienda que, para considerar el perfil ciudadano de la juventud universitaria actual, como el agente de cambio que requiere el presente y el futuro de la sociedad en México, deberá de trabajarse académicamente en su posibilidad de acción autónoma, para que pueda construir su futuro en lo individual y en lo colectivo con responsabilidad humana, pero siempre estando bien informada.

\section{Referencias Bibliográficas}

Álvarez Garro, L. (2014). El impacto de 30 años de reformas neoliberales en la percepción ciudadana de la relación entre democracia y bienestar humano en Costa Rica. Buenos Aires, Argentina: Consejo Latinoamericano de Ciencias Sociales [CLACSO].

Antón, J. (2012). El conservadurismo. En J. Antón, y Á. Rivero. Derechos y ciudadanía, pp. contrastes entre el liberalismo y el pensamiento conservador (pp. 11-26). México: Instituto Federal Electoral.

Artiles, M.; Martín, O.; Kappel, J.; Poliak, J.; Rebagliati, P. y Sánchez Bodas, A. R. (1994). Psicología humanista, pp. aportes y orientaciones. Buenos Aires: Editorial DOCENCIA.

Bernasconi, A. (2014). Autonomía universitaria en el siglo XXI, pp. nuevas formas de legitimidad ante las transformaciones del Estado y la sociedad. Páginas de Educación, 7, (2), 33-60.

Brunner, J. J. (2015). Transformaciones del espíritu comunitario de la universidad, pp. base de la responsabilidad social de la academia. En E. Aponte Hernández. La responsabilidad social de las universidades, pp. implicaciones para la América Latina y el Caribe (págs. 97-114). San Juan, Puerto Rico, pp. Instituto Internacional de la Unesco para la Educación Superior en América Latina y el Caribe [IESALC] [UNESCO].

Consejo Constitucional de la República Francesa. (1789). Declaración de los derechos del hombre y del ciudadano de 1789. París, Francia, pp. Consejo Constitucional de la República Francesa. Disponible en: https, pp.//www. conseil-constitutionnel.fr/es/declaracion-de-los-derechos-del-hombre-ydel-ciudadano-de-1789.

De la Cruz, S. (2012). La mercantilización de la educación. Revista Electrónica de Psicología Política. 9, (28), 48-54.

Fair, H. (2008). El sistema global neoliberal. Polis (Revista Latinoamericana) 21, 1-31. Disponible en: https: //doi.org/10.4067/S0718-65682008000200012 
Fernández González, N. (2017). La lucha simbólica por la educación en la globalización neoliberal. Revista Brasileira de Educação, 22, (71), 1-23. Disponible en: https: //doi.org/10.1590/s1413-24782017227171

Fernández, E. (2009). El sistema-mundo del capitalismo académico, pp. procesos de consolidación de la universidad emprendedora. Archivos Analíticos de Políticas Educativas, 17, 1-43.

Follari, R. A. (2009). Para un más allá de la mercantilización educativa, pp. ¿pueden administrarse los valores?. En P. Gentili, G. Frigotto, R. Leher, y F. Stubrin. Políticas de privatización, espacio público y educación en América Latina (págs. 163-180). Santa Fe, Argentina, pp. Consejo Latinoamericano de Ciencias Sociales [CLACSO].

García de la Mora, J. M. (1961). Werner Jaeger: filólogo, filósofo y humanista. Convivium, 11-12, 175-180.

Habermas, J. (1999). Problemas de legitimación en el capitalismo tardío. Madrid: Cátedra.

Hidalgo Villegas, L. N. (2017). Imaginario universitario, pp. ¿Qué significa la universidad para los jóvenes estudiantes en Guadalajara? Revista Pueblos y fronteras digital, 12, (23), 175-202. Disponible en: https: //doi.org/10.22201/ cimsur.18704115e.2017.23.292

Horrach Miralles, J. A. (2009). Sobre el concepto de ciudadanía: historia y modelos. Factótum 6, 1-22.

Iglesias, M. (2015). La continuidad del discurso neoconservador frente a la política exterior de la administración Obama. Revista UNISCI [Unidad de investigación sobre seguridad y cooperación] / UNISCI Journal, 38, 89-105. Disponible en: https: //doi.org/10.5209/rev_RUNI.2015.n38.49646

Jaramillo R, M. M. (2012). El principio de autonomía universitaria como autonomía democrática-descentrada, pp. antecedentes histórico-filosóficos de la evolución ético-política de su concepto. Revista de humanidades, 26, 195-247.

Landinelli, J. (2009). Las finalidades públicas de la universidad en el contexto de la globalización. En P. Gentili, G. Frigotto, R. Leher, y F. Stubrin. Políticas de privatización, espacio público y educación en América Latina (págs. 205224). Santa Fe, Argentina: Consejo Latinoamericano de Ciencias Sociales [CLACSO].

Massé Narváez, C. E. (2008). Autonomía estatal y universitaria, mercantilización del conocimiento y educación en el neoliberalismo. Educere, 12, (41), 387-395. 
Miñana Blasco, C., y Rodríguez, J. G. (2002). La educación en el contexto neoliberal, Universidad Nacional de Colombia. Disponible en: http: //www.humanas. unal.edu.co/red/files/3112/7248/4191/Artculos-eduneoliberal.pdf.

Monares, A. (2005). ¿Quién podría ser Neoliberal?. Polis (Revista Latinoamericana) $12,1-13$.

Olvera Rivero, A. (2008). Ciudadanía y democracia. México.: Instituto Federal Electoral [IFE].

Orden Jurídico Nacional. (2019). Constitución política de los Estados Unidos Mexicanos, pp. Publicado en el Diario Oficial de la Federación 5 de febrero de 1917. Última reforma publicada DOF 26-03-2019. (SEGOB, Ed.), pp. Dirección general de compilación y consulta del Orden Jurídico Nacional, pp. Disponible en: http: //www.ordenjuridico.gob.mx/Documentos/Federal/pdf/wo14166.pdf

Organización de las Naciones Unidas para la Educación, la Ciencia y la Cultura [UNESCO]. (1998). Conferencia Mundial sobre la Educación Superior en el siglo XXI: Visión y acción. París: Organización de las Naciones Unidas para la Educación, la Ciencia y la Cultura [UNESCO].

Ornelas Delgado, J. (2009). Neoliberalismo y capitalismo académico. En P. Gentili, G. Frigotto, R. Leher, y F. Stubrin. Políticas de privatización, espacio público y educación en América Latina (págs. 83-120). Santa Fe, Argentina: Consejo Latinoamericano de Ciencias Sociales [CLACSO].

Ortiz Gómez, M. G. (2014). El perfil del ciudadano neoliberal: la ciudadanía de la autogestión neoliberal. Sociológica, 29, (83), 165-200.

Pérez, S., y Castaño, R. (2016). Funciones de la universidad en el siglo XXI, pp. humanística, básica e integral. Revista Electrónica Universitaria de Formación del Profesorado, 19, (1), 191-199.

Rivero Franyutti, A. (2013). ¿Que son las humanidades y cuál ha sido su valor en la universidad? Revista de la Educación Superior, 167, (42), 82-100.

Salazar Olguín, H. D. (2018). Globalización, neoliberalismo y reforma educativa en México. Acoyauh, 59, 49-77.

Spinoza, B. (1986). Tratado político. Madrid: Alianza Editorial.

Tünnermann Bernheim, C. (2008). La autonomía universitaria en el contexto actual. Universidades, 36, 19-46.

Valverde Brenes, F. J. (2014). Persona o individuo en el (neo) liberalismo, pp. algunos fundamentos ideológicos. Pensamiento Actual. Universidad de Costa Rica, 14, (22), 29 - 41. 


\section{Vázquez Olivera, M. G. (2015). La calidad de la educación. Reformas educativas y control social en América Latina. Latinoamérica. Revista de Estudios La- tinoamericanos, 60, (C), 13-52. Disponible en: https: //doi.org/10.1016/j.la- rev.2014.10.001}

\section{Notas al final}

1 Tipo de artículo: Investigación científica autofinanciada por el autor sin apoyo institucional alguno; originado por interés y seguimiento de la línea de investigación del propio autor.

2 Arquitecto, Facultad de Arquitectura Universidad Autónoma de Nuevo León, México; Master en Administración, Facultad de Contaduría Pública y Administración (FACPYA), Universidad Autónoma de Nuevo León, México. Doctor en Filosofía con Orientación en Trabajo Social y Políticas Comparadas de Bienestar Social. Docente investigador de la Facultad de Contaduría Pública y Administración (FACPYA), Universidad Autónoma de Nuevo León, México. Dirección postal: Av. Pedro de Alba S/N, Ciudad Universitaria U.A.N.L., San Nicolás de los Garza, Nuevo León, México. Tel: 01 (81) 8329 4080. jorge.lealig@uanl.edu.mx Orcid: 0000-0002-5548-1943

3 Denominación utilizada para explicar la visión de una persona como una cosa inanimada. 